\title{
¿Apostamos desde el déficit o desde del conflicto? Visión relacional de un grupo de adicción al juego a través de la mentalización ${ }^{1}$
}

\author{
Ignacio Blasco Barrientos ${ }^{2}$ \\ IPR, ALYA (Madrid, España)
}

\begin{abstract}
En este trabajo se pretende trasmitir el punto de encuentro que en mi experiencia profesional ha tenido el trabajo de Killingmo y sus revisiones (Coderch) junto con el día a día de un grupo terapéutico de adicción al juego en el cual se trabaja desde perspectivas relacionales que invitan a entender los procesos de mentalización de los integrantes. Dos puntos clave para el desarrollo de esta comunicación son: 1) La orientación que el equipo terapéutico ha dado al grupo. Favoreciendo espacios de intercambio donde los integrantes han dejado ver su matriz relacional y capacidad de mentalización; 2) La evolución del juego en sus formas y espacios en los últimos años.
\end{abstract}

Palabras clave: Ludopatia, déficit, conflicto, adicción al juego, mentalización, relacional, transferencia.

This paper is intended to convey the meeting point in my professional experience has been the work of Killingmo and revisions (Coderch) together with the daily life of a therapeutic group of gambling. Group in which working from relational perspectives that invite you to know mentalization processes members. Two key points for the development of this communication are: 1) The guidance therapeutic team has given the group. Promoting exchange spaces where members have left see their relational matrix and mentalization process; 2 ) The evolution of gambling in its forms and spaces in recent years.

Key Words: gambling, deficit, conflict, mentalization, relational, transference.

English Title: Do we bet from the deficit or from the conflict? A Relational view of a gambling addiction group through mentalization.

\section{Cita bibliográfica / Reference citation:}

Blasco Barrientos, I. (2016). ¿Apostamos desde el deficit o desde el conflict? Una visión relacional de un grupo de adicción al juego a través de la mentalización. Clínica e Investigación Relacional, 10 (3): 711-722. [ISSN 1988-2939] [Recuperado de www.ceir.info] DOI: 10.21110/19882939.2016.1003

\footnotetext{
${ }^{1}$ Comunicación presentada en las IV Jornadas de Psicoanálisis Relacional que sobre el tema de la Mentalización, se desarrollaron en Salamanca los días 28 y 29 de Octubre de 2016.

${ }^{2}$ Psicoterapeuta individual y de grupo, miembro del Instituto de Psicoterapia Relacional. Dirección de contacto: i.blasco.barrientos@gmail.com

CeIR Vol. 10 (3) - Junio 2016 ISSN 1988-2939 - www.ceir.info

(c) Derechos reservados/Copyright de Clínica e investigación Relacional y los autores. Prohibida la reproducción total o parcial sin autorización expresa. Este material es para uso científico y profesional exclusivamente y puede contener información clínica sensible. Los editores no se responsabilizan de los contenidos de los autores. Dirigir las consultas sobre derechos y autorizaciones a ceir@psicoterapiarelacional.es
} 


\section{Breve revisión a las aportaciones de Killingmo:}

Killingmo de alguna manera desafía al psicoanálisis clásico tratando de integrar la psicología del déficit con la concepción clásica del conflicto. El trabajo pone el foco en ciertos pacientes a los que de alguna manera los señalamientos o interpretaciones psicoanalíticas no sólo no les ayudan a deshacer nudos sino que provocan reacciones contrarias (distanciamiento, desconexión, frialdad) dando lugar a análisis improductivos, fallidos o interminables.

En cambio observa como atendiendo a sutiles señales, ideas o fragmentos que el analizado va aportando puede colocarse de otra manera y por tanto intervenir en una dirección distinta a desenredar el conflicto entre sistemas o intrasistema. Estas señales son las que le llevan a ver fallas intrasistémicas, vacíos, faltas, huecos o espacios no nutridos. No existe tal conflicto ya que no hay estructura clara que la provoque, simplemente la estructura esta incompleta.

El resultado del análisis de Killingmo nos lleva a entender que ciertos pacientes no se benefician o no está en sus prioridades psíquicas el que sean interpretados sus conflictos, fantasías o sentimientos prohibidos. Al tener un Yo fragmentado, poco consistente, no del todo diferenciado, reciben este tipo de intervenciones de forma negativa, propiciando una distancia helada con su analista.

Esta fragmentación o vacío necesita ser, necesita ser afirmada, saber qué es. La pérdida de la propia identidad es la angustia que predomina y por tanto las intervenciones del analista deben ir en esa dirección. Afirmando la existencia, focalizar la relación, ponerlo en valor y validarlo como tal.

En palabras de Killingmo;

"La principal diferencia entre conflicto y déficit puede ser formulada en términos de representación del significado intencional. Así, el déficit hará referencia a una patología donde no existe una intencionalidad primaria. El yo ha sido dañado en un momento en que su capacidad para representar caudas y efectos así como su capacidad para experimentar el simismo como un centro estratégico no se ha desarrollado todavía. El resultado de esta falta de diferenciación del yo es un estado de confusión y sentimientos amorfos de vergüenza y culpa. Por tanto, en la patología del déficit no se trata de defenderse contra la angustia relacionada con las malas intenciones (fantasías, necesidades, sentimientos prohibidos) dirigidas al objeto como sucede en el conflicto. Contra lo que uno se defiende es fundamentalmente contra la angustia de fragmentación, es decir contra la perdida de la propia sensación de identidad.

Es necesario aclarar que esta diferenciación no es matemática, no es rígida. Killingmo mismo aclara la complejidad de dividir las patologías déficit y conflicto como dos compartimentos estancos explicando una suerte de continuo donde todo conflicto viene de cierto aspecto deficitario de una estructura así como todo déficit estructural grave puede devenir en conflicto en el marco terapéutico. Es la pericia y experiencia del terapeuta lo que entenderá 
si el paciente en un momento dado esta hablando desde la transferencia de déficit o de conflicto.

\section{Mentalización como apertura.}

El poder entender o captar desde donde nos habla un paciente será un trabajo que dependerá en gran medida de la experiencia del terapeuta, su intuición y sin duda alguna los procesos de mentalización que se den en el encuentro terapéutico. La vinculación de la mentalización con la formación del self es cada vez mayor. De ahí la importancia de hacer una lectura de los procesos mentales que nosotros como terapeutas tenemos y evidentemente, los de nuestro paciente. En palabras de Coderch "La mentalización es la manera natural que tienen los seres humanos de entenderse a símismos y entender a los otros"

Y está implícito en la definición que esa manera natural de entenderse esta sostenida por núcleos de experiencias de apego-relacionales. Estas experiencias-cimiento son las que nos colocan en posiciones deficitarias o de conflicto en según que momentos.

\section{Introducción del encuadre e historia del grupo terapéutico donde trabajo.}

Comencé en 2010 como observador-coterapeuta en una asociación de adicción al juego, el encuadre terapéutico consistía en un grupo abierto de un máximo de 9 pacientes, semanal. Cada 15 días venían junto a sus acompañantes (madres, hijos, mujeres o maridos). Coordinaba una psicóloga y había un coterapeuta (jugador rehabilitado). En aquella época el grupo estaba compuesto por jugadores de máquinas y bingo en su totalidad. El tratamiento tenia (y tiene) un fuerte componente cognitivo conductual, donde la prevención de respuesta, exposición o control de estímulos es parte de la columna vertebral del tratamiento. La duración del proceso terapéutico es de alrededor de dos años.

Lo que me llamó la atención era la interacción grupal, se dejaba poco espacio a la espontaneidad o debate, se negaban los emergentes, se hacia una especie de terapia individual en grupo.

Al ser casi mi primer contacto con grupos me pareció interesante pero en algún punto incómodo. Se podía palpar una tradición de grupos estilo doce pasos de Minnesota o jugadores anónimos donde la culpabilización, las voces altas y en ocasiones el insulto eran un recurso al que se tendía demasiado.

Estuve alrededor de un año y medio de observador, aprendí muchísimo, comprendí que esa dureza en cierta medida era necesaria, entendí lo que la adicción hace a la persona, o mejor dicho lo que deshace en la persona. Personas con fuertes valores, bondadosos y sensibles acababan fulminando el dinero del equipo de fútbol de su hijo en una mañana en el bar. Madres que de camino a comprar el pan se jugaban la nómina. 
También aprendí que mi estilo terapéutico no era ese ni lo seria, esa era una labor que podían hacer los coterapeutas (Personas rehabilitadas). Lo supe cuando tras esos dos años me ofrecieron coordinar y montar mi propio grupo. No rechacé la oportunidad.

A día de hoy llevo casi siete años trabajando en este ámbito, cuatro de ellos coordinando varios grupos. Junto a una buena compañera, Miriam y pacientes ya rehabilitados hemos creado ALYA (Asociación ludopatia y nuevas adicciones).

Mantenemos gran parte del encuadre: El control de estímulos y prevención de respuesta sigue siendo gran parte de la columna vertebral. Cuando hablo de control hablo de un control absoluto del dinero donde el paciente tiene que mancomunar su cuenta bancaria con su acompañante, donde ha de anular todas sus tarjetas de crédito y donde el primer año de tratamiento tiene que llevar siempre y sin acumular dos euros diarios (justificando diariamente cualquier gasto con un recibo).

El equipo está formado por: dos psicólogos y un observador (prácticas) así como una persona rehabilitada con una breve formación en dinámica grupal. La duración sigue siendo de dos años y el régimen del grupo es abierto. Acuden semanalmente los pacientes y quincenalmente con sus acompañantes.

Antes de meternos en materia señalar dos puntos clave que han propiciado el pensar sobre este trabajo.

El primer punto es, como he dicho antes la globalización del juego: la evolución tecnológica, la apertura legislativa y el ritmo social nos ha llevado a un punto donde el juego patológico es una red mucho más amplia que hace años, ha cambiado radicalmente el perfil del jugador. Actualmente este problema afecta transversalmente a toda la sociedad, hay un tipo de juego para casi cualquier edad, condición social y tipo de personalidad. Nos comenzaron a llegar pacientes mucho más jóvenes (ya no venían acompañados con las mujeres, sino con su padres, abuelas, hermanos).

Eran más jóvenes y la adicción se había fraguado mucho más rápido e intensamente, llevaban menos años jugando pero sus situaciones económicas y personales eran desastrosas.

El segundo punto es la apertura que mi equipo y yo comenzamos a facilitar en el grupo, a riesgo de que todo se descontrolase o que chocásemos con un techo ideológico o tradicional (miedo que estuvo presente durante mucho tiempo) intentamos favorecer la espontaneidad, recoger los emergentes, focalizar las emociones que surgían o intentar ponerles nombre. El clima fue cambiando, también la forma de trabajar del grupo, los vínculos ser fortalecieron, la horizontalidad comenzaba a aflorar, la comunicación fluía, en ocasiones la tarea del grupo se desviaba a puntos alejados del juego y sus consecuencias. Sorprendentemente ahí se fueron encontrando más los unos a los otros y favoreció de forma sustancial a la dinámica grupal. También mejoró algo que nos llamó mucho la atención: 
Favoreció a la aceptación de las normas (de gran dureza) de una forma mucho más natural y de mayor aceptación, era un mal compartido pero estaban todos en lo mismo, había que empujar conjuntamente.

En ningún momento olvidamos el por qué estábamos allí y los exhaustivos controles, simplemente facilitamos dentro de la rigidez del tratamiento espacios de reflexión profunda mediante el señalamiento, la interpretación de comentarios entre los participantes o mediante la apertura de debates surgidos de dudas individuales. El grupo en ocasiones era mucho más que la suma de sus partes, esto antes no ocurría, hasta aquel momento esa frase sólo la había leído en los libros. Se podría decir que el grupo se empoderó como tal,

El cambio de estilo nos llevó irremediablemente a trabajar no sólo de forma horizontal en el grupo, hablando sobre si se ha pensado o no en el juego, examinando al detalle si las cuentas económicas cuadran o no o recordando el daño que el jugador hizo en su momento. La apertura dio lugar a que floreciera la matriz relacional de cada componente, a traer al grupo historias, datos, recuerdos, detalles, sentimientos enquistados, culpas y perdones. Se profundizaba cada vez más en las relaciones de apego, en la historia relacional como conjunto de experiencias y vínculos, en definitiva estábamos facilitando el pensar sobre nuestros pensamientos surgiendo así la capacidad de mentalización de los integrantes junto con la valiosa información que ello conlleva.

Cuando acudían los acompañantes el trabajo se hacia aún más intenso, todo resonaba, el ambiente estaba muy caldeado, saltaban chispas, se lloraba, se reía. Irremediablemente esto nos daba al equipo terapéutico una información muy valiosa sobre cada uno de los integrantes, nos iluminaba caminos que confluían y resonaban, comenzábamos a entender qué les había llevado a consumirse en el juego.

Sorprendentemente ellos comenzaron a moverse psiquicamente hacia otros puntos, salían de la culpa y del castigo constante por haber malgastado tanto su vida y se planteaban qué les había llevado allí, qué les había hecho jugar de esta forma, qué les había separado de si mismos y sus familias. El clima grupal se cargaba de los patrones relacionales de cada uno de los integrantes, había mucho a lo que atender y la tarea ganaba en complejidad y contenido.

Con el tiempo he ido viendo cómo esas palabras del artículo de Killingmo tomaban sentido en el grupo de terapia, no sólo eso, me encontré que la evolución tecnológica del juego traía al grupo a personas con patrones algo distintos a lo que habitualmente se veía, esto provocaba situaciones de cierta complejidad y comencé a pensar cada vez más en ello.

\section{Killingmo entró al grupo}

Hemos ido viendo como cada integrante trae consigo su historia. Poco a poco fuimos observando el déficit o el conflicto en cada uno de ellos. Las formas de relacionarse, de 
analizar, de dar o nutrirse en el grupo arrojaban luz sobre lo que Killingmo desarrolló hace años. Curiosamente pude comprobar que esos dos perfiles solían confrontar entre si, se desconectaban, demandaban cosas distintas. Pude obervar otra interesante correlación, el juego problema que les había traído al grupo.

Vi como el jugador clásico de bar o bingo partía o se relacionaba transferencialmente desde el déficit, desde posiciones más depresivas: Desde el "me han quitado algo", "el mundo es injusto", "me ha faltado", "no me han dado"; Desde el vacío y desde la insatisfacción absoluta. El patrón intencional era muy representativo, había jugado, sí; había perdido mucho dinero, también. Pero no se reconocían frente a la máquina. Esto daba lugar a sentimientos extraños de vergüenza donde su intencionalidad quedaba relegada a un segundo plano, su angustia no era fruto de la culpa sino una angustia muy primaria, angustia al vacío. En algún punto no encontraban responsabilidad o causalidad directa en sus actos, no eran dueños de una parte de si mismos, había algo vacío, hueco.

Vacío que les desconectaba de si mismos y de sus problemas diarios, no enfrentaban nada, se refugiaban en el calor del acto compulsivo para después verse arrollados por una culpa dolorosísima e infinita que les fragmentaba pero a la vez les llevaba a volver al día siguiente a jugar. La máquina consiguió darles identidad, sólo eran algo cuando jugaban, al desconectarse de ella todo lo que se había evitado y negado aparecía como una pesadilla para volver a llevarles al pánico de que esa no era su vida.

Los "nuevos" jugadores venían de casinos, de apuestas deportivas, de las páginas de apuestas online. Curiosamente juegos de alguna forma más competitivos y donde el premio era mayor, también el riesgo. Como he dicho antes aparecían adicciones muy intensas desarrolladas en mucho menos tiempo, internet funciona las veinticuatro horas, los bares y bingos antes o después cierran y tienes que salir de casa para ir a ellos. En el trabajo hay conexión a internet, en casa, en el móvil, en la calle. La apuesta a una pulsación de distancia, no había espacio para la reflexión, pausa o el remordimiento, la satisfacción era instantánea.

Estas nuevas personalidades provocaron climas distintos a los que había que atender. Estos pacientes no les faltaba "nada", estaban perfectamente, habían perdido algo de dinero, nada más. Jamás olvidaré una frase de uno de ellos su primer día: " A mi los jugadores de máquinas me parecen unos gilipollas, no van a ganar nunca nada, están perdidos..." . Esta intervención muestra perfectamente el clima que de una forma $u$ otra se iba a repetir en multitud de sesiones. Estas personas se ponían por encima de los otros jugadores, les menospreciaban directa e indirectamente, al fin y al cabo pensaban que estaban en estructuras superiores y de alguna manera así fuimos observándolo. Sus movimientos internos venían de la competitividad con otros y contra sí mismos, no huían, se enfrentaban a si mismos patológicamente, no había una falta, había un revoltijo. Sus defensas eran mayores, las resistencias tensaban muchísimo el grupo. Curiosamente había ocasiones en las que llegaban a justificar su juego o incluso dudar de la terapia, aludiendo que ellos mismos serian 
capaces de dejarlo por si mismos, o peor aún, que serian capaces de jugar de forma controlada en un futuro, su identidad estaba muy marcada, había una clara intencionalidad y su dolor venia por la culpa, no por la angustia de fragmentación.

Estas diferencias entre ambos perfiles son muy marcadas desde la óptica de las intervenciones de unos sobre otros. Pacientes conflicto se refugiaban en defensas evasivas, intelectualizadas. Esto provocaba ataques de los pacientes déficit que exigían ser entendidos en sus dramas y que los otros aceptasen los suyos propios. Los pacientes conflicto rehuían de ellos, argumentaban desde el "pasa página". Unos no soportaban la "debilidad", los otros el descaro de la evitación. Unos se fundían en emociones, demandaban sujeción a los coordinadores, los otros negaban sus frustraciones, las proyectaban en la debilidad del otro, evitaban y desafiaban al coordinador.

\section{La intencionalidad primaria y transferencias surgidas.}

La intencionalidad del acto tiene mucho peso en esta diferenciación que nos ayudará a entender no sólo la transferencia que puedan ofrecer los pacientes déficit o conflicto sino su relación con la adicción. El paciente conflicto ha sido dañado estructuralmente cuando el Yo aun no estaba formado, la relación causa - efecto no ha sido del todo integrada; por ello la transferencia ha de ir orientada a la comprensión, a acompañar en el significado, a estar ahí. En cambio el paciente conflicto tiene un Yo estructurado y una intencionalidad consciente, por ello la tarea transferencial irá encaminada a trabajar conjuntamente para hacer frente a los impulsos dañinos y no ceder ante ellos.

Pondré un pequeño ejemplo que muestra a la perfección esta distinción: " La fantasía de recuperar el dinero perdido: jugando".

De sobra son conocidas las ideas irracionales fruto de una adicción, la más idiosincrática es sin duda alguna la fantasía de recuperación, la idea de ser capaz de recuperar todo lo perdido en un golpe de suerte y así poder tapar todos los agujeros económicos y problemas causados. Hay una importante diferencia entre jugadores de máquinas o bingo donde el premio más cuantioso no supera los seis mil euros en el mejor (peor) de los casos. En cambio jugadores de apuestas deportivas $u$ online en una sola apuesta pueden (y por tanto creen poder) llevarse una cuantía mucho mayor.

Este detalle por insignificante que parezca ha dado lugar a infinitas discusiones en el grupo, los pacientes por conflicto saben que pueden acertar una apuesta y ganarlo todo, confían en ellos mismos, se animan pensando en que antes o después ganarán, sus causas tienen efecto. Hay una "estrategia" que suele fallar pero esto les hace culparse menos. Existe un yo estratégico.

Pacientes déficit no tienen un yo estratégico que les lleve a pensar que recuperarán todo lo perdido, vuelven a la máquina con tal de evitar la angustia interna, y ya en la máquina piensan 
en recuperar lo perdido en ese momento, saben que la máquina les llena, consiguen apartar la angustia pero no hay una intención de recuperar todo lo perdido ya que no es posible.

En este punto se han dado fuertes discusiones, faltas de entendimiento ya que detrás de todo esto el paciente conflicto esta convencido que es más inteligente que sus compañeros deficitarios y que estos son unos perdedores, ironías de la vida.

Transferencia de conflicto y transferencia déficit (La intencionalidad del acto).

Killingmo hace esta diferenciación entre transferencia de conflicto y de déficit. En la transferencia de conflicto encontramos los impulsos y afectos dirigidos hacia las representaciones internalizadas de los objetos emocionalmente significativos mientras que en la de déficit se expresan necesidades dirigidas hacia objetos que no han sido internalizados.

Esta definición nos ayuda a ver los aspectos estructurales completos e incompletos del paciente dentro de una transferencia propia del psicoanálisis más clásico. Por ello veo necesario complementarla con la definición que nos ofrece Coderch en Pluralidad y diálogo en psicoanálisis (2006): "La transferencia es la manera como el analizado organiza la situación analítica de recuerdo con la totalidad de sus experiencias pasadas, tanto conscientes como inconscientes, ya sean estas últimas las propias del inconsciente reprimido o las que constituyen el inconsciente no reprimido o de procedimiento".

Sin duda alguna esta definición es mucho más cercana a las posiciones desde donde trabajamos y sin lugar a duda hay una fuerte relación con la capacidad de mentalización ya que la manera en que el sujeto organiza su pensamiento es fruto de todas las experiencias que desde sus primeras relaciones de apego ha ido sintiendo, integrando y entendiendo en base a si mismo y los otros. Por ello a nivel grupal, el abanico de transferencias que entre entre sus integrantes se vayan dando estarán fuertemente ligados a los procesos de mentalización de los mismos. Como terapeuta poder verlo desde esa óptica ha facilitado el desarrollo de las bases de este trabajo.

Me centraré algo más en la transferencia que a lo largo del tratamiento los diferentes pacientes iban mostrando con el grupo. Haciendo una especial diferenciación entre pacientes conflicto y déficit desde el momento que se les hace la primera entrevista hasta su evolución en el grupo.

Los pacientes más clásicos, jugadores de máquina y bingo venían el primer día descompuestos, las primeras entrevistas eran muy duras para ellos. Veías el drama en primera persona y su necesidad de cambio, no entendían nada, esto no era nuevo para ellos, desde sus partes más deficitarias no encontraban sentido estructural a lo que les había llevado delante de mi. Sólo sentían angustia. Veían en el terapeuta a su "salvador", alguien 
que por fin les iba a sacar del agujero donde ellos se habían metido y en definitiva llenar ese vacío.

Era muy explícita esa entrega, a veces resultaba incómoda ya que de alguna forma parecía que ellos no tenían que hacer nada en el tratamiento, por fin habían llegado a un sitio donde se les iba a sacar del juego y recuperar una vida normal, la pastilla mágica que todo lo cura, signo inequívoco de la falta de intencionalidad que Killingmo resalta.

Este detalle ya deja entrever posibles daños estructurales o posiciones primarias donde se les ha de solucionar el problema o donde la herramienta para el cambio la tiene el terapeuta y no ellos mismos.

El comienzo en el grupo del paciente déficit solía ser relativamente tranquilo, parcos en palabras aceptaban silenciosamente las normas, mostraban una aparente conformidad con la dureza de los primeros días. El grupo en un primer momento sustentaba el vacío y dolor de muchos años de juego y el malestar que en su núcleo familiar se vivía. Las necesidades de sujeción y comprensión eran cubiertas por el grupo, se nutrían de ello.

En cuanto iba pasando el tiempo el cambio era notable, sus necesidades y prioridades empezaban a cambiar. Las normas de control se hacían más duras y las ganas de jugar (conscientes o no) empezaban a asomar la cabeza. Los primeros conflictos aparecían cuando las necesidades ya no eran cubiertas por completo en el grupo, surgía el sentimiento de que estaban siendo castigados, el dolor causado y dinero perdido a lo largo de los años pasaba a segundo plano. Comenzaban a pedir (exigir) privilegios, se rebelaban contra las normas, contra los compañeros y contra el terapeuta. Se palpaba ese sentimiento de "el mundo me debe algo", "no me merezco esto", "no soy nadie, me tenéis anulado". El no se hacia responsable de lo que le estaba pasando, era el grupo quien se lo imponía de manera injusta, como un castigo sin saber el delito cometido.

Ante este tipo de intervenciones la respuesta del grupo muchas veces iba encaminada a la reprimenda, a recordar todo lo que había dañado, a culparle o a hacerle ver que esas salidas de tono podían tener que ver con un síndrome de abstinencia o incluso con estar ocultando algo (créditos, recaídas, deudas no declaradas). Esto aumentaba el malestar del paciente así como el tono de sus intervenciones y el calor de la sala, momentos de mucha tensión. Digamos que estas intervenciones buscaban el por qué, se encaminaban a interpretar los sentimientos de ansiedad del paciente, eran intervenciones interpretativas.

Otro tipo de intervenciones (las afirmativas) serian las de comprensión y entendimiento, respuestas orientadas a validar la experiencia que estaba teniendo, tomarla como normal y pedirle que aceptase la dureza del tratamiento, que con el tiempo iría viendo y viviendo los beneficios de hacerlo. En este punto ayudaban muchos los pacientes más veteranos que no solo explicaban con claridad esto sino que se ponían como ejemplo. El resultado de estas intervenciones era mucho más positivo. 
En pacientes conflicto, pacientes generalmente más jóvenes donde su juego problema suele ser el juego online y derivados la entrada al grupo solía ser completamente distinta, como antes he mencionado ellos venían con la teoría aprendida, sabían que habían hecho algo mal pero también entendían que en sus manos estaba su recuperación. No eran tan conscientes de la adicción que les había llevado allí, le restaban importancia e incluso afirmaban no ser adictos. Con este perfil es de imaginar el primer contacto conmigo, ponían en duda todo lo que se les explicaba, negaban haber perdido el control, desafiaban ante cualquier intento de acercamiento.

Algunos no llegaban a entrar al grupo, no en ese momento, semanas o meses después volvían tras haber perdido una suma aún más importante de dinero.

Cómo ya expliqué antes este perfil intentaba separarse psicológicamente de los anteriores, no aceptaban que fuesen como ellos, no conectaba con quien se lamentaba o se redimía de una forma primaria, menos aún si se machacaban. Estaban en el mismo grupo pero a años luz de distancia, al menos al principio, en las primeras sesiones.

Los conflictos con este tipo de pacientes surgían cuando el grupo les instaba a reconocer las partes más crudas de su adicción, cuando le pedían que asumiese su ludopatia para comenzar a cambiar, estos no lo aceptaban, los ludópatas eran los otros. Esto puede chocar en un principio con la idea de intencionalidad primaria ya que parece que el no aceptar el problema es no aceptar la causa-efecto de sus actos. No es exactamente así, el paciente conflicto sabe que se ha pasado, que en algún momento ha perdido el control pero eso no conlleva a que su Yo pueda hacer frente a la adicción, es decir lucha internamente contra sus tendencias adictivas y en cierto momento cree que puede contra ellas. Su yo esta estructurado y en ocasiones sobrevalorado.

La transferencia que ofrece este perfil es más agresiva, la queja pasa a un segundo plano frente a la confrontación y el desafío. Por ello es llamativo cómo las intervenciones afirmativas orientadas a la comprensión, la aceptación o el intento de entendimiento no surten mucho efecto sino lo contrario. En cambio intervenciones de tinte más interpretativo donde simplemente se señala o se le busca sentido a las confrontaciones que en el grupo vuelca el paciente favorecen la reflexión y en ocasiones facilitan la capacidad de insight.

Es importante señalar que a nivel teórico es posible diferenciar de una forma más fina y elegante estos dos perfiles de paciente. En la práctica esta diferenciación es más ardua y compleja. Tal como he señalado es a lo largo de la primera entrevista y los primeros meses en el grupo donde es más plausible la diferencia. Pero como el propio Killingmo afirma no han de entenderse como compartimentos estancos. 


\section{Conclusión:}

El trabajo resume mi experiencia profesional en un ámbito muy diferenciado de la clínica clásica individual donde paciente y terapeuta se relacionan bidireccionalmente. Dentro de la idiosincrasia de las adicciones y dentro de un encuadre grupal he intentado trasmitir cómo dos perfiles de pacientes en dos categorías distintas (Jugador clásico / paciente déficit VS Jugador online / conflicto) confluyen de una forma muy interesante.

Observación fraguada a lo largo de los años gracias a la orientación del grupo que ha permitido volcar la matriz relacional y la capacidad de mentalización de cada uno de los integrantes, facilitando el proceso de análisis individual de los pacientes por parte de los terapeutas; dando lugar esto a una mayor comprensión del problema a tratar así como mejores herramientas de intervención.

Lo que Killingmo expone y Coderch afina en relación a estos tipos de paciente se hace patente en que:

Pacientes deficitarios con una falla estructural importante tienden de forma patológica a llenarse o evitar ese vacío con la conducta compulsiva y repetitiva de juego en máquinas tragaperras.

Pacientes conflicto en cambio vehiculizan sus problemas intrasistema y conflictos con sus objetos internos en juegos más agresivos y con una mayor percepción de ganancia así como de peligro como las apuestas deportivas, euromillones o juegos online.

Entre ellos se ponen en juego transferencias propias de cada perfil desconectándose unos de otros en un primer momento y reconectándose gracias a la convivencia grupal y las intervenciones afirmativas o interpretativas que el terapeuta va dando y el grupo integrando.

En definitiva, más allá de transferencias, déficitis y conflictos el fin último de una terapia de grupo con adictos (sea lo que sea) es la abstinencia y rehabilitación completa de quien demanda ayuda. Si además se trabaja en grupo una meta es la fluidez en la dinámica y su capacidad de sanar por sí mismo. Por ello si algo hay que resaltar más allá de la argumentación teórica es el buen trabajo del grupo y sus integrantes donde finalmente el conflicto resonó en el déficit y el déficit entró en conflicto.

No quiero cerrar este articulo sin mencionar expresamente a ALYA, asociación de la cual soy fundador junto con un grupo de personas maravillosas y que sin ella no seria posible nada de esto; Si de algo estoy seguro es que mientras se trabajaba en el grupo este artículo estaba siendo escrito; así como mientras escribo esto estamos empezando la siguiente sesión grupal. 


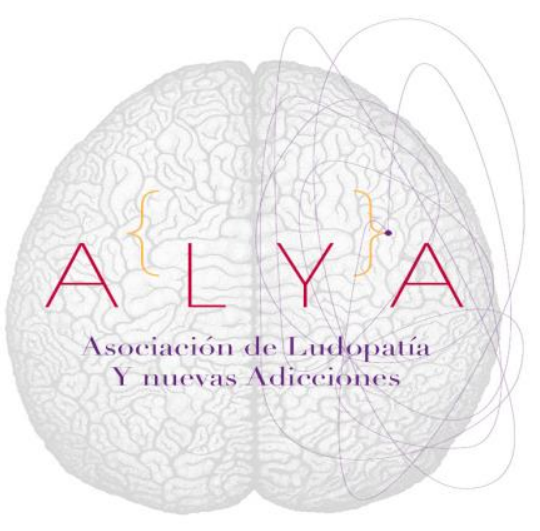

\section{REFERENCIAS}

Ávila. A (2013) . La tradición interpersonal. Perspectiva social y cultural en psicoanálisis. Madrid: Ágora Relacional.

Coderch, J. (2007). Conflicto, déficit y defecto. Clínica e investigación relacional, 1(2): 259-371.

Coderch, J. (2014). Avances en psicoterapia relacional. Nuevos campos de exploración para el psicoanálisis. Madrid: Ágora Relacional.

Echeburua, E., Becoña, E. y Labrador, F.J.; Fundación GAUDIUM (2010). El juego patológico: Avances en la clínica y tratamiento. Madrid: PIRAMIDE

Fonagy, Peter. (2000). Aspectos patológicos y acción terapéutica. Aperturas psicoanalíticas no 4 . Garrido, Miguel; Jaén Pedro y Domínguez Ana (2004). Ludopatia y relaciones familiares: clínica y tratamiento. Barcelona: PAIDOS

Guimón, J. (2003). Manual de terapias de grupo: Tipos, modelos y programas. Madrid: Biblioteca Nueva.

Killingmo,B (1989). Conflict and Deficit. Implications for technique. Int j. Psycho-anal. 70:65-79. Traducción castellana revisada por GRITA.

Original recibido con fecha: 2-10-2016 Revisado: 29-10-2016 Aceptado: 31-10-2016 